Methods: The website was designed with support of the Dutch Alzheimer Society in three focus group session with persons with dementia and their spouses. In these sessions existing websites, which had older users are a target group were discussed. Themes discussed were about the design of the website as use of colors, legibility and usability. Based on this, a demonstration website was made and discussed and judged by the participants. Results: A total of six older adults participated in these focus group sessions. Participants prefer a website which is easy to print, navigation should be on maximal three levels Menu of the website should be on the left site of the page. Participants dislike the use of photographs in order to increase ambiance and pictograms, because these do not provide any additional value to the information given. Conclusions: Participants prefer a website to be accessible just as reading a book. The results were partial in accordance with the results reported in the literature for web design for persons with cognitive disabilities. The results in this report concern the design of the website only. This design process was executed in a single experiment. Therefore, the findings should be interpreted with caution. Further study is scheduled to examine the usefulness of the designed website.

\section{P2-406 MEASURING OF THE ONSET OF ALZHEIMER'S DISEASE AND OTHER COGNITIVE DEMENTIAS IN SAMPLE SURVEYS: IDENTIFYING EARLY SIGNS OF IMPAIRMENT AND THE IMPACTS OF PROXY RESPONSES ON FOLLOW UP INTERVIEWS}

\begin{abstract}
James McNally, University of Michigan, Ann Arbor, Michigan, United
\end{abstract} States.

Background: Increasingly, longitudinal studies on the aging life course seek to measure a multitude of factors, from health to family structure to economic stability. While Alzheimer's remains a relatively rare outcome in the general population, the diagnosis of Alzheimer's or other significant dementia represent a common reason for refusals in follow up surveys of the aged. Even if such individuals are followed their responses are generally obtained through proxy respondents whom may or may not have intimate knowledge of the impacted individual. Even with large sample populations, the focus and design of a survey can impact the likelihood of following individuals with cognitive impairments through time and the types of questions asked regarding cognitive functioning. It is presently unknown if these differences impact observed results in a statistically significant manner as the diagnosis of Alzheimer's is something that occurs within the follow up stream and rarely represents an initial observation health status. This paper looks at available longitudinal studies which identify respondents diagnosed with Alzheimer's or other cognitive impairments and seeks to categorize patterns in response rates, refusals and follow up interviews. Methods: This paper builds on earlier work reviewing the progress of cognitive measurement in sample surveys. The paper begins with a brief review of the development of data measuring cognitive function since the 1960's. The paper then focuses on multi-wave longitudinal studies of the aged who follow respondents until they leave the survey due to death or health related refusal. Drawing largely from seminal studies including the NLTCS, the HRS, ADAMS, and the LSOA I and II the paper establishes a context of evolving understanding of the measurement of cognition occurs in large surveys. More importantly, by comparing those respondents ultimately diagnosed with cognition issues to those who do not report these problems, we seek to identify early indicators of cognitive impairment that may be predictive of the terminal diagnosis. This is done through observed changes in the use of standard tests of cognition but also by examination of response patterns across time; frequency of inconsistent answers, patterns of refusal, don't know and no response, as well as the presence of proxy respondents. The survey instrument itself has often been identified as a source of mental stress for some respondents in studies of the elderly and it is argued that those with greater problems in completing a survey should be at higher risk of subsequent refusal due to cognitive health concerns. What is poorly understood however if there are observable patterns in non-response that are predictive of early signs of declines in cognitive functioning. Results: As would be expected measured declines in cognitive function and overall health across time are predictive of respondents later dropping out of surveys due to cognitive impairment. Often, however, these changes are missed either due to issues such as the lack of detailed probing into cognitive functioning, extended periods between follow-up interviews, and the potential conditioning of respondents due to repeated administration of similar questions. When examining response patterns we find more subtle indicators of changes in memory, ability to perform complex tasks and the inability to consistently answer specific questions. Questionnaire series regarding employment history, income and wealth, health history and mobility represent area where memory and cognitive functioning can result in inconsistent answers across waves or increased levels of missing information. These differences while speculative at this time do suggest that questionnaires could be designed to increase the sensitivity of such patterns and that they could be tested for validity of concept. If more fully developed these kinds of approaches might represent a less intrusive diagnostic tool for early cognition concerns that could be then pursued with more precise instruments and tests. Conclusions: The future of research into the early diagnosis of cognitive impairment seems to be moving in new directions, particularly in the development of lifecourse studies that follow individuals from their youth onward. Our capacity to develop life course studies that measure the aging process from cradle to grave is improving but much work and decades of research lay before us. Until such a time we need to continue to seek new and innovative ways to use studies such as the HRS, the NLTCS and the earlierLSOA surveys. This paper has shown one potential direction we may follow by seeking commonalities in behaviors and response patterns among respondents prior to a diagnosis of Alzheimer's or other dementia that typically leads to their dropping out of the survey population. This analysis approach may help us better understand who is at risk of cognitive impairment and offer further understanding in patterns of behavior that are suggestive of this risk.

\section{P2-407 EARLY INDICATORS OF DEMENTIA RISKS: ESTS OF COGNITION AND ACHIEVEMENT AMONG THE 1960 PROJECT TALENT HIGH SCHOOL COHORT}

James McNally ${ }^{1}$, Martha Sayre ${ }^{1},{ }^{1}$ University of Michigan, Ann Arbor, Michigan, United States.

Background: Project TALENT is the single largest source of information on the life experiences of students who were in high school in 1960. In the spring of I960, more than 400,000 students were given a two-day battery of tests and inventories. The test battery was designed to provide baseline data on the aptitudes and abilities of these individuals, as well as to assess their interests and various personal characteristics. Methods: Project TALENT data files include thousands of data points about the backgrounds, abilities, interests, goals, opinions, educational experiences, and career outcomes of a large and representative sample of adults who are now in their 60 s. The battery included cognitive tests in the following areas: abilities, knowledge, and achievement; dispositional traits; interests; and background characteristics. Results: In the 50 years since the 12 th graders were first tested, a wealth of original research has emerged from the analysis of Project TALENT across diverse scientific fields. Project TALENT allows us to examine the impacts on ability in great detail and across multiple ethnic and SES groups. Similarly, reasoning ability and creativity are central in one's cognitive retention in later life. In addition to testing simple knowledge of facts, the TALENT battery included tests scores for a wide range of basic cognitive abilities. In the 50 years since the 12th graders were first tested, a wealth of original research has emerged from the analysis of Project TALENT across diverse scientific fields. We seek to continue this process, extending the study to examination of how these early life experiences affected the later life outcomes. Conclusions: Project TALENT overcomes many if not all of the barriers faced by other studies that might be transitioned into life course studies of cognitive development and change including small sample size, limited generalizability, and sample follow-up. Because of the established value and the ongoing research interest in Project TALENT, we argue that it is critical to increase the accessibility of this collection to an emerging generation of social science researchers. 
Project TALENT Data Structure

\begin{tabular}{|c|c|c|c|}
\hline Sample & Original date file name & Number of Records & Unit \\
\hline \multicolumn{4}{|c|}{ Mailer Files-all students for whom an address was updated in 1978} \\
\hline 12th grade & MAIL12_UP0478.dat & 85,498 student & 300 \\
\hline 11th grade & MAIL11_UP0478.dat & 99,695 student & 300 \\
\hline 10th grade & MAIL10_UP0478.dat & 108,338 student & 300 \\
\hline 9th grade & MAIL9_UP0478.dat & 111,928 student & 300 \\
\hline \multicolumn{4}{|l|}{ Grade Level Files - Probability sample student data } \\
\hline 12th grade & TAL12_00.dat & 81,130 student & 2390 \\
\hline 11th grade & TAL11_00.dat & 92,419 student & 2390 \\
\hline 10th grade & TAL10_00.dat & 99,574 student & 2390 \\
\hline 9th grade & TAL9_00.dat & 103,893 student & 2390 \\
\hline 1\% Public Use File & pubuse.dat & 4,000 student & 2383 \\
\hline $4 \%$ Item Level File & fourpent.dat & 13,478 student & 2432 \\
\hline General School Sample File & gsc.dat & 1,236 student & 1540 \\
\hline Nonprobability sample (Knox Country and 15 -year olds) & not yet found & 34,360 student & 2383 \\
\hline 1963 Retest sample & not yet found & 7542 student & 4565 \\
\hline
\end{tabular}

+ Not applicable.

Notes: A-No documentation available;B Code book available in PDF format only,D-Limited documentation available;E-Code book available in PDF format only.

P2-408 CRITICAL ANALYSIS OF ROAD TEST DESIGNS FOR OLDER DRIVERS WITH COGNITIVE IMPAIRMENT

Brian Ott ${ }^{1}$, George Papandonatos ${ }^{1}$, Peggy Barco ${ }^{2}$, Timothy Souza ${ }^{3}$, Jennifer Davis ${ }^{1}$, David Carr ${ }^{2},{ }^{1}$ Brown University, Providence, Rhode Island, United States; ${ }^{2}$ Washington University, St. Louis, Missouri, United States; ${ }^{3}$ ABC/ACE Driving School, Brooklyn, Connecticut, United States.

Background: The goals of this study are to critically review and analyze currently employed road test designs from published research studies involving cognitively impaired older drivers in order to define potential improvements that would enhance reliability, accuracy and validity. Methods: A literature review is complemented by detailed analyses of selected road test studies from Brown and Washington Universities. We examine 1) inter-rater reliability of driving instructors from different geographic regions, 2) weighting bias by driving instructors in forming global impressions, 3 ) weighting of individual road test items by the instructors in forming global impressions, and 4) comparisons of road test and naturalistic home driving components using factor analysis. Results: Driving performance scores for videotaped home driving epochs by two driving instructors were modestly correlated. Differences in global impressions were partially attributable to the items of driving ability that were emphasized. Road test scores were unidimensional and reflected by a major factor related to awareness of signage and traffic behavior, while naturalistic home driving reflected two factors related to lane keeping as well as traffic behavior. Conclusions: Despite differences in geography and local driving regulations and practices, we have demonstrated that road test courses and driving examination scales can be harmonized; however, refinement in this area is still needed. Further improvements could include development of nationwide standards for scoring methodology for driving examiners, as well as road courses that incorporate the special challenges to examining the older driver in a more naturalistic and valid way.

\section{P2-409 ASSISTIVE TECHNOLOGIES FOR OLDER ADULTS WITH ALZHEIMER'S DISEASE: THE LUSAGE USER-LAB EXPERIENCE}

Maribel Pino ${ }^{1}$, Melodie Boulay ${ }^{1}$, Grégory Legouverneur ${ }^{1}$, Ya Huei $\mathrm{Wu}^{1}$, Victoria Cristancho-Lacroix ${ }^{1}$, Anne-Sophie Rigaud ${ }^{1},{ }^{1}$ Hopital Broca, Paris, France.

Background: Over the past years, a number of assistive technology (AT) products have been conceived and developed to support independent living and quality of life of individuals with Alzheimer's disease (AD) and their caregivers. Some mainstream technological products can be also used for these purposes. However, little experimental research has been done on usability issues concerning these end-users. We describe the goals of LUSAGE, a usability laboratory specialized in elderly individuals with cognitive impairment. We present a general methodological framework for usability testing with this population and illustrate it with three studies: (a) Prototype software for cognitive training, (b) A graphic interface of an assistive robot, (d) Two sport video-games. After highlighting key findings and limitations we suggest future research directions in this field. Methods: Studies. The aim of these studies was to assess if the current product was usable by target users without major assistance and to expose all their major usability deficiencies. Target group. Older adults with mild-to-moderate AD. Procedure. The software and the video games user-test consisted of: One exploratory session and four test-sessions. Assessment of the robot's graphic interface was made in a single session. Interface manipulation and facial expression were video recorded. Quantitative and qualitative performance and preference measures were collected. Results: Broadly participants could use the three systems with different levels of guidance. When conducting multiple test-sessions we noticed a general improvement in performance measures. Usability assessment allowed us to identify specific ergonomic deficiencies of each product. Our results confirm that not only specific cognitive loss but also behavioral, psychological and sensorial deficits influence the way individuals with AD interact with technological products. Cognitive functioning, disability level and socioeconomic background may vary a lot from one individual to another. Due to the heterogeneity of AD presentation it seems complicated to define one single profile that fits all potential users. Conclusions: Planning, designing and conceiving technologies to support $\mathrm{AD}$ patients and their caregivers require end-user involvement throughout all the development lifecycle. Usability assessment in laboratories specialized in this audience constitute an innovative enterprise. However further work is required for the development of adapted assessment methodologies.

P2-410

EVALUATION OF BENEFITS OF A HEALING
GARDEN FOR ALZHEIMER PATIENTS:
NEW METHODOLOGY TO EVALUATE
A DESIGN CONCEPT INTEGRATING ARTISTIC
AND NEUROPSYCHOLOGICAL APPROACHES

Therese Rivasseau Jonveaux ${ }^{1}$, Manon Yzoard $^{2}$, Reinhard Fescharek ${ }^{3}$, Alina Pop ${ }^{4}$, Christel Jacob ${ }^{4}$, Martine Batt ${ }^{2}$, Laetitia Demarche ${ }^{4}$, Laure Soulon ${ }^{4}$, Gabriel Malerba ${ }^{4}$, Alain Trognon ${ }^{2},{ }^{1}$ CHU Nancy, Nancy, France; ${ }^{2}$ Université Nancy 2, Psychologie, Nancy, France; ${ }^{3}$ Fescharek Sculpture \& Design, Marburg Lahn, Germany; ${ }^{4}$ Centre Paul Spillmann CHU Nancy, Nancy, France. 